\title{
Ataxia-Telangiectasia and Refractory Peripheral T-cell Lymphoma: Considerations for Therapy and Role of Bone Marrow Transplantation
}

\author{
Saara Kaviany ${ }^{1}$, Carrie Kitko ${ }^{1}$, Cesar Rueda ${ }^{2}$, Roshini Abraham ${ }^{2}$, Debra Friedman ${ }^{1}$, Sarah \\ Neumann $^{1}$, Becky Manes ${ }^{1}$, Sara Zarnegar-Lumley ${ }^{1}$, and James Connelly ${ }^{1}$ \\ ${ }^{1}$ Monroe Carell Junior Children's Hospital at Vanderbilt \\ ${ }^{2}$ Nationwide Children's Hospital
}

October 1, 2020

\begin{abstract}
Certain patients with inborn errors of immunity have defects in DNA damage response, predisposing them to malignancy. Subsequent cancer therapy may require substantial attenuation given defective DNA repair; however, this carries risk of incomplete disease control. We describe a 5-year-old boy with peripheral T-Cell lymphoma with ataxia-telangiectasia (A-T). After incomplete chemotherapeutic response, he underwent allogenic hematopoietic cell transplantation (allo-HCT) with an attenuated preparative regimen, but developed graft rejection and relapse. Following remission with salvage chemotherapy, second allo-HCT with reduced intensity conditioning (RIC) resulted in minimal toxicity and short-term disease control. HCT with RIC can be considered in patients with A-T.
\end{abstract}

\section{Introduction}

Ataxia-telangiectasia (A-T) is an autosomal recessive disorder caused by pathogenic variants in ATM, which is critical for double-stranded DNA break repair. Bi-allelic variants lead to progressive cerebellar dysfunction, immunodeficiency, oculocutaneous telangiectasias, and an increased risk of cancer, specifically lymphoid ${ }^{1}$. Peripheral T-Cell Lymphoma-Not Otherwise Specified (PTCL-NOS), a predominantly nodal mature T-cell lymphoma, is rare in children ${ }^{2}$. Treatment in children is not uniform, but reported outcomes are poor, with a 5 -year event free survival of $59 \%^{3}$. Hematopoietic cell transplant (HCT) is considered a viable salvage option, with the majority receiving allo-HCT.

Treatment planning for patients with inborn errors of immunity (IEI) with impaired DNA damage response (DDR) has to accommodate this critical limitation to aggressive approaches. Patients with A-T have demonstrated worsening ataxia with vinca alkaloids, increased hemorrhagic cystitis (including late-onset) with alkylators, and declining lung function with bleomycin ${ }^{4-7}$. Furthermore, patients with A-T are at higher infection risk due to their intrinsic immunodeficiency ${ }^{4-7}$. HCT is rarely employed in A-T with limited reports of survival ${ }^{8}$. Herein, we discuss a pediatric patient with A-T and PTCL-NOS who underwent a second HCT following a tailored conditioning regimen and experienced minimal transplant-related toxicity to provide a framework for transplant consideration.

Results

The patient presented at age five with fevers, diffuse lymphadenopathy, and no prior diagnosis of A-T. An excisional lymph node biopsy revealed CD30 ${ }^{\text {neg }}$ PTCL-NOS, and bone marrow (BM) involvement classified him with Stage IV disease ${ }^{9}$. He was treated with dose adjusted-EPOCH (etoposide, prednisone, vincristine, cyclophosphamide, doxorubicin ${ }^{10}$ ). Interim-restaging demonstrated clinical and radiographic remission, with 
no morphologic evidence of lymphoma in BM; however, molecular residual disease was detected by polymerase chain reaction of rearrangement of T-cell receptor (TCR) gamma chain leading to HCT evaluation.

In early childhood the patient developed lower extremity weakness prompting a neurology evaluation, including a non-informative brain magnetic resonance imaging (MRI). Increased drooling, decreased grip, and wobbly gait out of proportion to expected toxicities, and diagnosis of a rare lymphoma at a young age led to evaluation for predisposing conditions. Targeted genetic sequencing revealed compound heterozygous variants in trans: (1) a pathogenic variant (c.2921+1G>A) and (2) a variant of uncertain significance (VUS) (c.8041G $>$ A; p.V2681M) in ATM . To establish the functional significance of the variants, a flow cytometric assay assessing DNA repair defects demonstrated completely absent ATM phosphorylation along with decreased gamma-H2AX (Figs. 1A and 1B), one hour after induction of DNA double-strand breaks, which is typically when maximal phosphorylation is expected ${ }^{11-13}$. This result revealed a functional defect in the ATM protein, indicating that the VUS is pathogenic. A Western immunoblot revealed absent ATM protein (not shown). These results confirmed a diagnosis of A-T, and further dose-escalation of EPOCH was halted.

Consideration of myeloablative autologous transplant was abandoned for a minimally-intensive allo-HCT. The patient received a matched unrelated donor HCT (MUD-HCT) following "Fanconi-style" preparative regimen of anti-thymocyte globulin (ATG), fludarabine and very low dose busulfan (estimated cumulative area under the curve (AUC) exposure: $1.9 \mathrm{mg}^{*} \mathrm{~h} / \mathrm{L}$ ). Unfortunately, the patient had primary graft failure with autologous reconstitution. His BM evaluation at day +100 was negative for residual disease by morphology; however, TCR-gamma chain rearrangement was detectable. On day +147 he presented with lymphadenopathy and biopsy-proven recurrence. In conjunction with family preference, we pursued a curative approach based on the anaplastic large cell lymphoma (ALCL)-99 backbone ${ }^{14}$ (methotrexate, ifosfamide, cytarabine, etoposide, dexamethasone, doxorubicin). Enhanced supportive care measures included mesna and hydration with alkylators, and methylene blue with ifosfamide to mitigate mucosal and neurologic toxicities, respectively. No dose reductions were made given previous tolerance and the refractory nature of his disease. Following two cycles, disease evaluation demonstrated clinical, radiographic and molecular remission.

Our patient received a MUD-HCT from a different donor with reduced intensity fludarabine and busulfan (estimated cumulative AUC: $57.3 \mathrm{mg}$ h/L). He had minimal toxicity including grade II mucositis, neutrophil engraftment on day +13 and discharge home on day +18 . His day +30 marrow demonstrated $100 \%$ donor myeloid engraftment with no residual lymphoma but persistent recipient T-cells (38\% donor). The patient had no graft-vs-host disease (GvHD) and tacrolimus taper was initiated at day +58 . He had improving peripheral blood T-cell chimerism on day +86 (100\% donor myeloid cells, $60 \%$ donor T-cells.) He developed no post-engraftment adverse events until day +94 when he presented with headache and diplopia, revealing lymphomatous involvement of his left optic nerve and cerebral spinal fluid consistent with PTCL-NOS relapse. He was also noted to have Epstein-Barr virus (EBV) viremia (41,200 copies/mL), presumed to be primary EBV as he had no documentation of prior infection. The family chose symptomatic management with dexamethasone and hospice enrollment for end-of-life care.

\section{Discussion}

Patients with A-T have a lifetime cancer incidence of $10-20 \%^{15}$. Dose reduction of chemotherapy may be considered, based on the premise that cancer cells may have increased susceptibility due to germline defects in DNA repair ${ }^{16}$. The role of HCT is not well established. HCT in pediatric cancer typically employs myeloablative dosing of DNA-damaging chemotherapy to reduce disease burden and permit engraftment which can cause severe toxicity and higher grade $\mathrm{GvHD}^{17}$ in patients with A-T. It remains unclear if hematopoietic cells in A-T have increased sensitivity to conditioning agents.

In patients with A-T, minimal intensity "Fanconi-based" conditioning has been the most accepted ${ }^{8}$. Slack et. al reported survival without GvHD in only two of eight patients with A-T following HCT after modified "Fanconi conditioning" without GvHD. Of the 6 patients who died, $67 \%$ experienced grade 2-4 GvHD despite well-matched donors and myeloablative conditioning in 5. Mortality causes included GvHD, multiorgan failure, viral re-activation or post-transplant lymphoproliferative disorder (PTLD). The underlying 
defective repair mechanisms may predispose to GvHD, as demonstrated in patients with Fanconi anemia and dyskeratosis congenita ${ }^{18}$.

Mixed chimerism seen in modified German Fanconi protocols, allowed for autologous hematopoietic cells to coexist with allogeneic hematopoiesis ${ }^{19}$. Remaining recipient A-T hematopoietic cells with prior exposure to conditioning agents have reduced capacity to correct genotoxic stress, and thus higher theoretical risk of secondary malignancies.

The rate of PTLD appears higher in post-HCT patients with $\mathrm{A}-\mathrm{T}^{14}$ and may be due to the role of ATM in the EBV life cycle. In the EBV lytic cycle, ATM recruits DDR proteins to viral double-stranded linear DNA to promote viral replication ${ }^{18}$. Less is known about ATM in latency, but ATM limits early hyperproliferation of infected B-cells in vitro, which may inhibit expression of viral oncogenes and promote the EBV latency program away from lymphoma and PTLD. Loss of ATM function may therefore directly contribute to a higher incidence of EBV-driven lymphoma and PTLD in patients with A-T. As our patient did not have complete lymphoid replacement, EBV reactivation may have been driven by recipient B-cell infection and impaired regulation of B-cell hyperproliferation. Full donor lymphoid engraftment may prevent post-transplant viral reactivation in recipient cells reducing mortality.

We propose that RIC be preferred when balancing risk of graft failure, disease relapse and risks imposed by residual recipient chimerism including secondary myeloid malignancy, recipient-derived PTLD, and defects in immune reconstitution. While our patient ultimately had disease relapse and EBV infection, we wish to highlight the challenges in treating aggressive disease in this cohort and suggest consideration of alternative therapeutic strategies to potentially improve outcomes.

Conflict of Interest Statement:

There are no affiliations that are relevant and important with any organization that to any author's knowledge has a direct interest, particularly a financial interest, in the subject matter discussed.

Acknowledgements

Research reported in this publication was supported by the Department of Health and Human Services National Institutes of Health National Cancer Institute under award number 5K12 CA090625-21. The assessment of the DNA repair defect was supported by the Department of Pathology and Laboratory Medicine, Nationwide Children's Hospital, Columbus.

We also acknowledge Laura Hall PharmD and Sarah Timaeus PharmD for their input on therapeutic and supportive care regimens.

\section{References}

1. Roohi J, Crowe J, Loredan D, et al. New diagnosis of atypical ataxia-telangiectasia in a 17-year-old boy with T-cell acute lymphoblastic leukemia and a novel ATM mutation. J Hum Genet .

(2017) 62(5) 581-584

2. Gordon BG, Warkentin PI, Weisenburger DD, et al. Bone marrow transplantation for peripheral T-cell lymphoma in children and adolescents. Blood . (1992) 80(11) 2938-2942

3. Sorge C, Costa LJ, Taub JW, S. Cairo M, Xavier AC. Incidence and outcomes of rare paediatric nonhodgkin lymphomas. Br J Haematol . Published online 2019. doi:10.1111/bjh.15194

4. Abadir R, Hakami N. Ataxia telangiectasia with cancer. An indication for reduced radiotherapy and chemotherapy doses. Br J Radiol .

(1983) 56(665) 343-345

5. Mann JR. Proceedings: A patient with ataxia-telangiectasia showing abnormal radiosensitivity to normal doses of irradiation. Br J Radiol . (1976) 49(582) 560 
6. Sandlund JT, Hudson MM, Kennedy W, Onciu M, Kastan MB. Pilot study of modified LMB-based therapy for children with ataxia-telangiectasia and advanced stage high grade mature B-cell malignancies. Pediatr Blood Cancer. (2014) 61(2) 360-362

7. Sandoval C, Swift M. Treatment of lymphoid malignancies in patients with ataxia- telangiectasia. Med Pediatr Oncol . (1998) 31(6) 491-497

8. Wolska-Kuśnierz B, Gennery AR. Hematopoietic Stem Cell Transplantation for DNA Double Strand Breakage Repair Disorders.Front Pediatr . Published online 2020. doi:10.3389/fped.2019.00557

9. Rosolen A, Perkins SL, Pinkerton CR, et al. Revised international pediatric non-Hodgkin lymphoma staging system. J Clin Oncol . (2015) 33(18) 2112-2118

10. Wilson WH, Bryant G, Bates S, et al. EPOCH chemotherapy: Toxicity and efficacy in relapsed and refractory non-Hodgkin's lymphoma. J Clin Oncol . (1993) 11(8) 1573-1582

11. Buchbinder D, Smith MJ, Kawahara M, Cowan MJ, Buzby JS, Abraham RS. Application of a radiosensitivity flow assay in a patient with DNA ligase 4 deficiency. Blood Adv . (2018) 2(15) 1828-1832

12. Cousin MA, Smith MJ, Sigafoos AN, et al. Utility of DNA, RNA, Protein, and Functional Approaches to Solve Cryptic Immunodeficiencies.J Clin Immunol . (2018) 38(3) 307-319

13. Barmettler S, Coffey K, Smith MJ, et al. Functional Confirmation of DNA Repair Defect in Ataxia Telangiectasia (AT) Infants Identified by Newborn Screening for Severe Combined Immunodeficiency (NBS SCID).J Allergy Clin Immunol Pract . Published online 2020. doi:10.1016/j.jaip.2020.08.008

14. Wrobel G, Mauguen A, Rosolen A, et al. Safety assessment of intensive induction therapy in childhood anaplastic large cell lymphoma: Report of the ALCL99 randomised trial. Pediatr Blood Cancer .

(2011) 56(7) 1071-1077

15. Suarez F, Mahlaoui N, Canioni D, et al. Incidence, presentation, and prognosis of malignancies in ataxia-telangiectasia: A report from the french national registry of primary immune deficiencies. $J$ Clin Oncol . (2015) 33(2) 202-208

16. Seidemann K, Henze G, Beck JD, et al. Non-Hodgkin's lymphoma in pediatric patients with chromosomal breakage syndromes (AT and NBS): Experience from the BFM trials. In: Annals of Oncology . ; 2000. doi:10.1093/annonc/11.suppl_1.S141

17. Slack J, Albert MH, Balashov D, et al. Outcome of hematopoietic cell transplantation for DNA doublestrand break repair disorders. J Allergy Clin Immunol . (2018) 141(1) 322-328.e10

18. Gadalla SM, Sales-Bonfim C, Carreras J, et al. Outcomes of Allogeneic Hematopoietic cell transplantation in patients with Dyskeratosis Congenita. Biol Blood Marrow Transplant .

(2013) 19(8) 1238-1243

19. Ussowicz M, Wawrzyniak-Dzierżek E, Mielcarek-Siedziuk M, et al. Allogeneic Stem Cell Transplantation after Fanconi Anemia Conditioning in Children with Ataxia-Telangiectasia Results in Stable T Cell Engraftment and Lack of Infections despite Mixed Chimerism. Biol Blood Marrow Transplant . (2018) 24(11) 2245-2249

Figure Legend:

Figure 1A and 1B. Flow cytometric analysis of the DNA damage response (DDR) in A-T. Peripheral blood mononuclear cells (PBMCs) from a healthy control and the patient were rested overnight and then either subjected to low-dose irradiation (2Gy, IR) or were not irradiated (unirradiated control, non-IR), and the phosphorylation of ATM (pATM) and the histone, H2AX (gH2AX) was assessed in T, B and NK cells at $1 \mathrm{~h}$ (top panel) and $24 \mathrm{~h}$ (bottom panel) post-irradiation with appropriate unirradiated controls for each time point. In contrast to the healthy control, the patient T, B and NK cells do not demonstrate 
pATM at $1 \mathrm{~h}$ or $24 \mathrm{~h}$ post-irradiation. Since H2AX is phosphorylated by several kinases, including ATM, there is some level of residual $\mathrm{gH} 2 \mathrm{AX}$ detected (median fluorescence intensity -MFI- ratio of irradiated to unirradiated) in the patient lymphocyte subsets, though it is lower than the healthy control, especially for $\mathrm{T}$ and NK cells at $1 \mathrm{~h}$ post-irradiation, and more importantly, the MFI ratio does not show the normal kinetics of dephosphorylation at $24 \mathrm{~h}$ post-irradiation, like the healthy control. The frequency (\%) of T, B and NK cells expressed as a delta value (irradiated - unirradiated) at $1 \mathrm{~h}$ post-irradiation is decreased for $\mathrm{gH} 2 \mathrm{AX}$ in $\mathrm{T}$ cells, and NK cells compared to the control, but not in B cells.

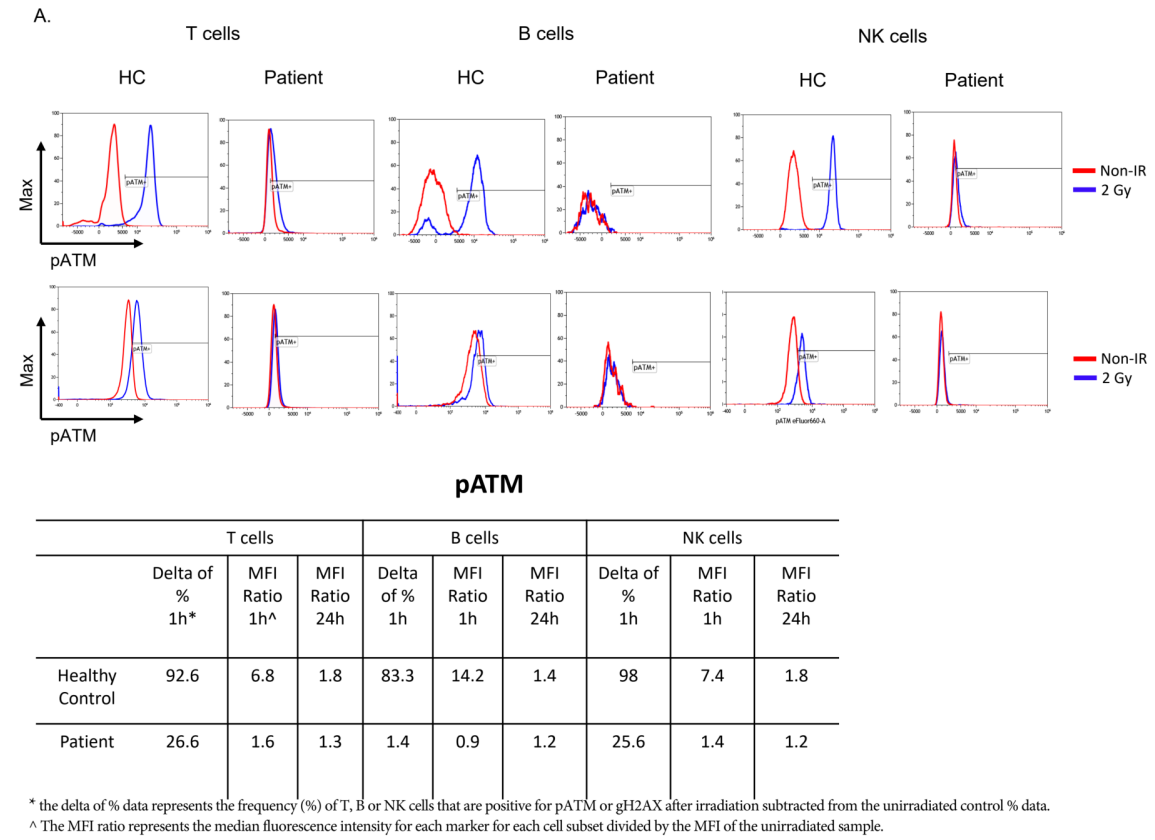

